\title{
Identification of conserved and novel mature miRNAs in selected crops as future targets for metabolic engineering
}

\author{
Fatima Noor ${ }^{1}$, Rahma Alshamrani², Munazza Gull ${ }^{3}$, Muhammad Aamer Mehmood1 ${ }^{1}$, Sidra Aslam ${ }^{*}$ \\ ${ }^{1}$ Department of Bioinformatics and Biotechnology, Government College University, Faisalabad, Pakistan \\ ${ }^{2}$ Department of Biological Sciences, King Abdulaziz University, Jeddha, Saudi Arabia \\ ${ }^{3}$ Biochemistry Department, King Abdulaziz University, Jeddha, Saudi Arabia
}



This is an Open Access article distributed under the terms of the Creative Commons Attribution 3.0 License. (https://creativecommons.org/licenses/by/3.0), which permits unrestricted use, distribution, and reproduction in any medium, provided the original work is properly cited.

\section{Introduction}

Energy has become the basic necessity in the social and economic development as well as in improving the standards of human being. Since 1970, researchers have paid a great deal of attention towards the development of technologies using renewable resource of energy in the context of high energy crises
(Gokcol et al., 2009). Among various sources, biomass from agricultural practices (bagasse, wheat/rice straw), wastewater cultivated microalgae (Shahid et al., 2020) and biomass from non-arable lands has shown promising potential as a renewable and lowcost feedstock to produce energy either biological or thermochemical processes (Mehmood et al., 2017, Ahmad et al., 2017, Ye et al., 2018). Thermochemical 
methods have shown dominance over biological methods in terms of robustness, efficiency, and costeffectiveness (Mehmood et al., 2019). Besides, regardless of the method of choice, higher biomass productivity is one of the desired parameters to enhance the cost-effectiveness of the bioenergy production. Bioenergy crops are considered as an auspicious source of the renewable energy (Sims et al., 2006). Biogas, ethanol and biodiesel are leading bioenergy products with respect to modern bioenergy (Yuan et al., 2008). The utilization of bioenergy is particularly high in the countries good financial backing or tax incentives, as for example China, Sweden, and Brazil (Wright, 2006).

Various approaches have been adopted to enhance the biomass productivity of the biomass including agricultural management practices to metabolic engineering for the subsequent use of biomass to produce bioenergy. Among various targets of metabolic engineering, Micro-RNAs have come forward as targets of interest due to their diverse roles in plant physiology including biomass production (Joshi et al., 2017). MicroRNAs (miRNA) are small non-coding RNAs, comprising about 22 nucleotides (Zhang et al., 2006b) and perform diverse physiological roles in the development of plant ;(Nogueira et al., 2007, Chitwood et al., 2009, RubioSomoza et al., 2009), abiotic and biotic stress responses (Shukla et al., 2008, Ruiz-Ferrer and Voinnet, 2009), signal transduction, protein degradation (Guo et al., 2005, Zhang et al., 2006b), post-transcriptional gene expression, and cellular metabolism (Zhang et al., 2006b, Zhao et al., 2010). The miRNA sequences of monocotyledonous and dicotyledonous plants are available in the miRBase. The identification of novel miRNA is a preliminary step to figure out the evolution of miRNAs in plant species along with their role in plant physiology. Hence, may lead to cultivation of the selected crop plants under salt/drought stress, after modifying the stress-responsive metabolic pathways.

A large number of miRNA have been reported in Glycine max (756), Oryza sativa (738), Zea mays (325), Sorghum bicolor (241), Brassica napus (92), Triticum aestivum (125), in miRBase (http://www.mirbase.org/) (Griffiths-Jones et al., 2007). The present study was focused on the identification and phylogenetics-based molecular characterization of conserved mature miRNAs in the aforementioned crops. The miRNA families have been shown to regulate physiological processes in the studied crops under stress conditions. Hence, study was aimed to propose the targets, those can be used in future for the metabolic engineering as well as in biomass production to meet the need of energy crises in future

\section{Material and Methods}

\section{Retrieval of miRNA data}

Plant genome contains hundreds of miRNAs. A very limited data is available for miRNA. In the current study, miRNA data of bioenergy was retrieved from miRBase database for further analysis (Figure 1). The miRBase database is considered as one of the main storehouses to collect miRNA genes since its inception because it provides a user-friendly interface offering a detailed overview of miRNA of interest and includes mature miRNA sequence along with their genomic coordinates and gene family. The dataset of the mature miRNAs sequences of Glycine $\max$ (no=756 mature), Oryza sativa (no=738), Zea mays (no=325, mature), Sorghum bicolor $(\mathrm{no}=241)$, Brassica napus $(\mathrm{no}=92$ mature), and Triticum aestivum (no=125 mature) were collected from miRBase.

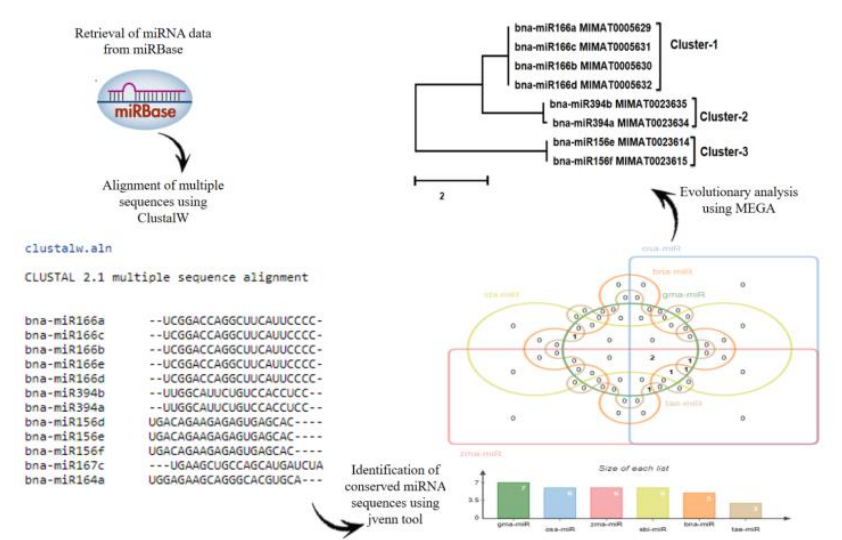

Figure-1. Schematic diagram representing the overall methodology used for the identification of conserved miRNA sequences in bioenergy crops

\section{Multiple sequence alignment using ClustalW}

The miRNA sequences of the bioenergy crops were then subjected to ClustalW for multiple sequence alignment (Chenna, 2003), which is a freely available and a frequently-used tool for the alignment of multiple sequences. It works on the basis of progressive alignment method. Therefore, it was used to reveal the conserved consensus by performing multiple alignments among mature miRNA sequence. 


\section{Phylogenetic analysis using MEGA}

The phylogenetic analyses of aligned sequences were carried out by utilizing Molecular Evolutionary Genetics Analysis (MEGA). Due to its user-friendly interface and availability of multiple methods for phylogenetic tree building, MEGA is well known tool for evolutionary analysis. MEGA offers the comparative analysis of aligned sequences. In order to infer the evolutionary history among aligned sequences, the Neighbor-Joining method was used. The Maximum Composite Likelihood method was employed to calculate the evolutionary distances. The scale tree is drawn with branch length in the same units as those of the evolutionary distances used to infer the phylogenetic tree.

\section{Identification of conserved miRNA}

After performing alignment, a separate phylogenetic tree was constructed for each bioenergy crop. Because some conserved miRNA along with their corresponding gene family were found in more than one bioenergy crop, therefore Jvenn tool (http://jvenn.toulouse.inra.fr) was used to identify conserved miRNA (Bardou et al., 2014) among the selected bioenergy crops.

\section{Results}

\section{Identification of intra-specific homologous mature miRNA sequences}

On the basis of sequence identity and phylogenetic relationship, the homologous sequences in Glycine max were clustered (Figure 2). The present analysis was comprised of 13 nucleotide sequences. These sequences were further subjected to MEGA for phylogenetic analysis. From pairwise deletion option, all uncertain positions were deleted. The final dataset contained total of 26 positions and which were grouped into 6 clusters. All the miRNA sequences were shown belong to a cluster that showed $100 \%$ sequence identity and their associated genomic coordinates (Table 1). Likewise, a phylogenetic model was employed in Oryza sativa with the aim of identifying all identical miRNA sequence (Figure 3). The analysis was comprised of 20 different nucleotide sequences where final datasets were shown to contain 23 positions which grouped into 6 clusters. All miRNA sequences were shown to belong to a cluster that showed $100 \%$ sequence identity and their associated genomic coordinates (Table 2). Similarly, the same phylogenetic model was employed in Zea mays to identify identical miRNA sequence (Figure 4). The analysis comprised of 14 nucleotide sequences and the final dataset contained 23 positions and have been grouped into 5 clusters. In this case, all the miRNA sequences were shown to belong to a cluster that showed $100 \%$ sequence identity and their associated genomic coordinates (Table 3). Same phylogenetic model was employed in Sorghum bicolor where analysis comprised of 14 nucleotide sequences (Figure 5 ) and the final dataset had a total of 21 positions and were grouped into 6 clusters. The miRNA sequences which belonged to a cluster that showed $100 \%$ sequence identity and their associated genomic coordinates are shown in Table 4. The phylogenetic analyses of Brassica napus contained 8 nucleotide sequences where final dataset had total of 24 positions and were grouped into 3 clusters (Figure 6). The miRNA sequences which belonged to a cluster that showed $100 \%$ sequence identity and their associated genomic coordinates are shown in Table 5.

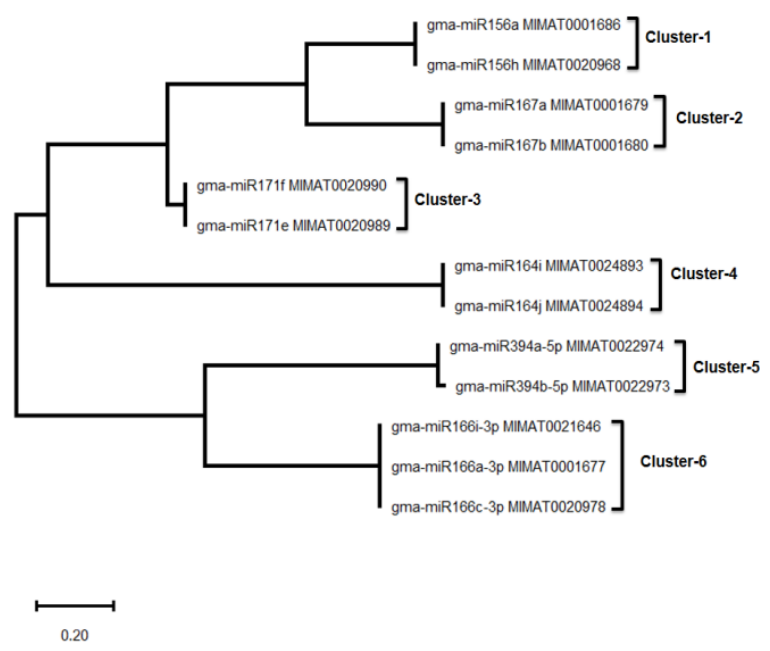

Figure-2. Evolutionary relationship in Glycine max. This analysis involved 13 nucleotide sequences. 


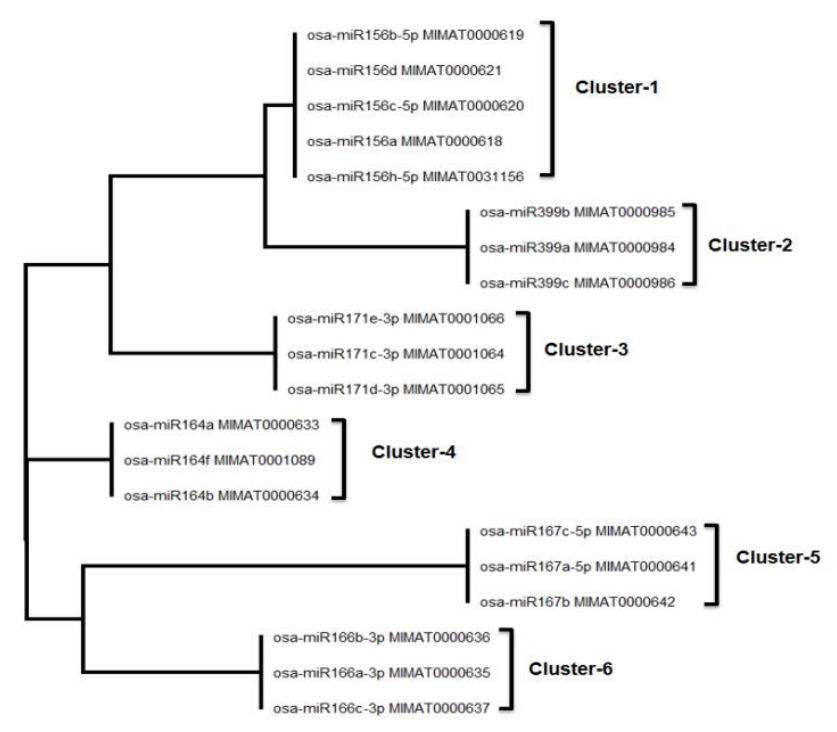

$\longmapsto$

Figure-3. Representation of evolutionary relationship in Oryza sativa based on 20 nucleotide sequences.

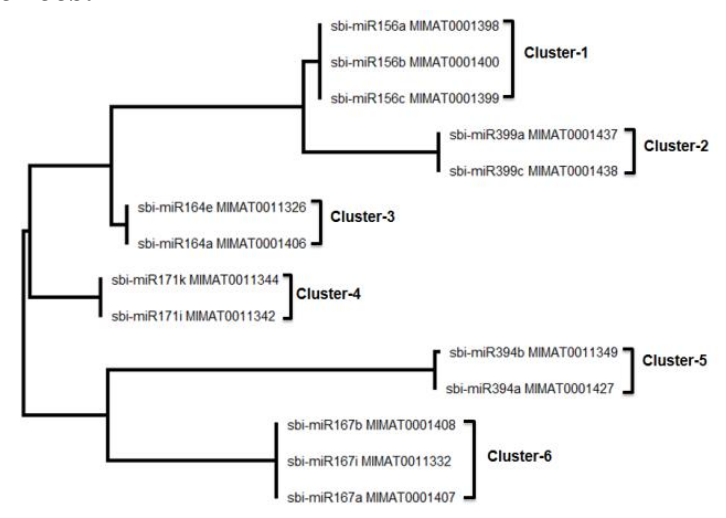

Figure-4. Representation of evolutionary relationship in Zea mays based on 14 nucleotide sequences.

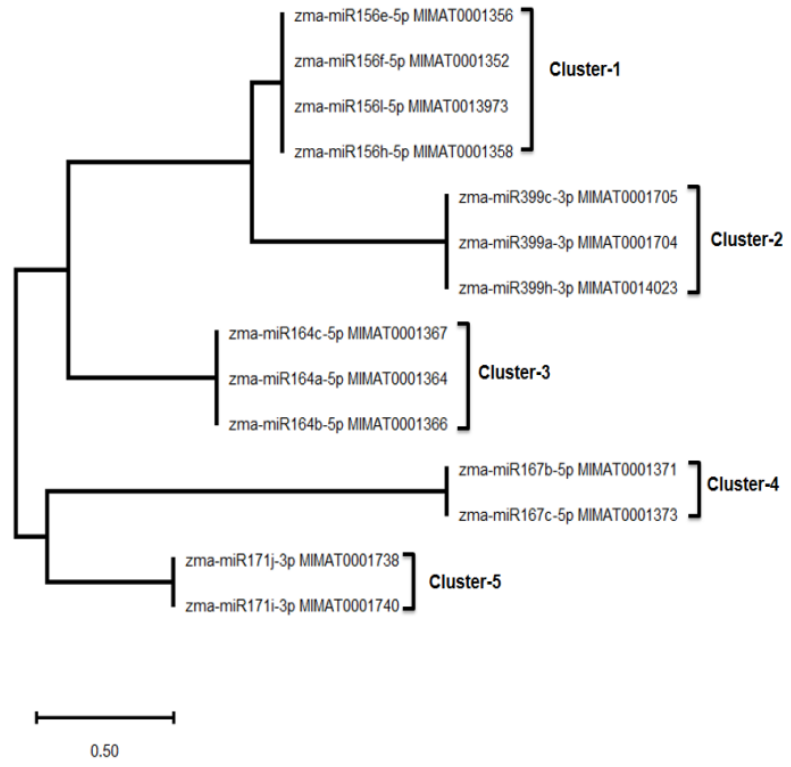

Figure-5. Representation of evolutionary relationship in Sorghum bicolor based on 14 nucleotide sequences.
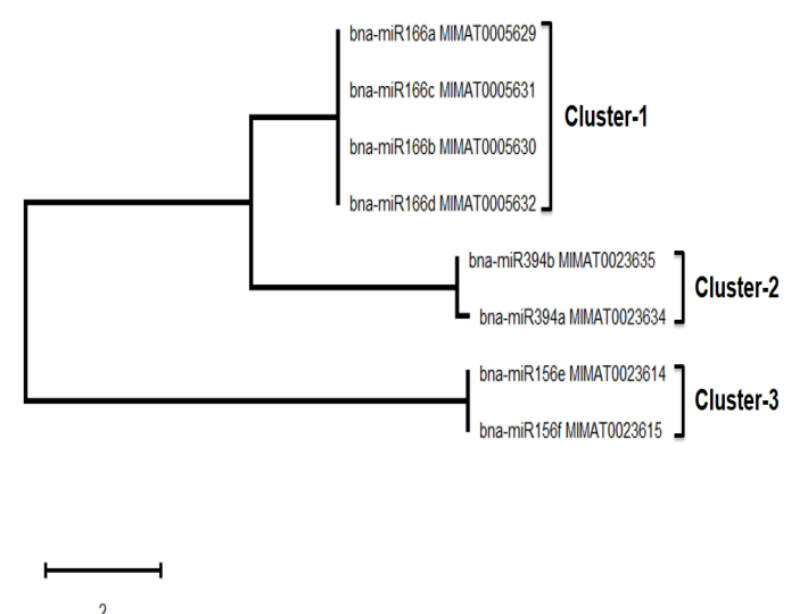

Figure-6. Evolutionary relationship in Brassica napus based on 8 nucleotide sequences. 
Fatima Noor et al.

Table-1. Summary of miRNAs grouped in clusters along with the members, genomic coordinates, gene families and the mature miRNA sequences in Glycine max

\begin{tabular}{|c|c|c|c|c|}
\hline Sr. No. & Members & Gene family name & Genomic coordinates & Mature miRNA sequences \\
\hline \multirow{2}{*}{ Cluster 1} & $\begin{array}{l}\text { gma-miR156a } \\
\text { MIMAT0001686 }\end{array}$ & \multirow{2}{*}{$\begin{array}{l}\text { MIPF0000008; } \\
\quad \text { MIR156 }\end{array}$} & chr17: 5885507-5885628 [-] & \multirow[t]{2}{*}{ ugacagaagagagugagcac } \\
\hline & $\begin{array}{l}\text { gma-miR156h } \\
\text { MIMAT0020968 }\end{array}$ & & chr4: 5047205-5047294 [-] & \\
\hline \multirow{2}{*}{ Cluster 2} & $\begin{array}{l}\text { gma-miR167a } \\
\text { MIMAT0001679 }\end{array}$ & \multirow{2}{*}{$\begin{array}{l}\text { MIPF0000023; } \\
\text { MIR167_1 }\end{array}$} & chr19: 42073549-42073667 [+] & \multirow{2}{*}{ ugaagcugccagcaugaucua } \\
\hline & \begin{tabular}{|l|} 
gma-miR167b \\
MIMAT0001680
\end{tabular} & & chr2: 14838068-14838188 [-] & \\
\hline \multirow{2}{*}{ Cluster 3} & $\begin{array}{l}\text { gma-miR171f } \\
\text { MIMAT0020990 }\end{array}$ & \multirow{2}{*}{$\begin{array}{l}\text { MIPF0000030; } \\
\text { MIR171_1 }\end{array}$} & chr15: 8505075-8505164 [-] & \multirow{2}{*}{ ugauugagccgugccaauauc } \\
\hline & $\begin{array}{l}\text { gma-miR171e } \\
\text { MIMAT0020989 }\end{array}$ & & chr5: 38773113-38773202 [-] & \\
\hline \multirow{2}{*}{ Cluster 4} & \begin{tabular}{|l|} 
gma-miR164i \\
MIMAT0024893
\end{tabular} & \multirow{2}{*}{$\begin{array}{l}\text { MIPF0000045; } \\
\text { MIR164 }\end{array}$} & chr18: 49506475-49506631 [+] & \multirow[b]{2}{*}{ uggagaagcagggcacgugca } \\
\hline & \begin{tabular}{|l|} 
gma-miR164j \\
MIMAT0024894 \\
\end{tabular} & & chr19: 48278744-48278831 [+] & \\
\hline \multirow{2}{*}{ Cluster 5} & $\begin{array}{l}\text { gma-miR394a-5p } \\
\text { MIMAT0022974 }\end{array}$ & \multirow[b]{2}{*}{$\begin{array}{l}\text { MIPF0000100; } \\
\text { MIR394 }\end{array}$} & $\operatorname{chr} 17: 41517111-41517267[+]$ & \multirow[t]{2}{*}{ uuggcauucuguccaccucc } \\
\hline & $\begin{array}{l}\text { gma-miR394b-5p } \\
\text { MIMAT0022973 }\end{array}$ & & chr14: 48784355-48784458 [+] & \\
\hline \multirow{3}{*}{ Cluster 6} & $\begin{array}{l}\text { gma-miR166i-3p } \\
\text { MIMAT0021646 }\end{array}$ & \multirow{3}{*}{$\begin{array}{l}\text { MIPF0000004; } \\
\text { MIR166 }\end{array}$} & chr2: $14544488-14544584[+]$ & \multirow{3}{*}{ ucggaccaggcuucauucccc } \\
\hline & $\begin{array}{l}\text { gma-miR166a-3p } \\
\text { MIMAT0001677 }\end{array}$ & & chr16: 1934911-1935056 [-] & \\
\hline & $\begin{array}{l}\text { gma-miR166c-3p } \\
\text { MIMAT0020978 }\end{array}$ & & chr7: 4502830-4502959 [-] & \\
\hline
\end{tabular}

Table-2. Summary of miRNAs grouped in clusters along with the members, genomic coordinates, gene families and the mature miRNA sequences in Oryza sativa

\begin{tabular}{|c|c|c|c|c|}
\hline Sr. No. & Members & $\begin{array}{l}\text { Gene family } \\
\text { name }\end{array}$ & Genomic coordinates & Mature miRNA sequences \\
\hline \multirow{5}{*}{ Cluster 1} & $\begin{array}{l}\text { osa-miR156b-5p } \\
\text { MIMAT0000619 }\end{array}$ & \multirow{5}{*}{$\begin{array}{l}\text { MIPF0000008; } \\
\quad \text { MIR156 }\end{array}$} & Chr1: 4666341-4666516 [+] & \multirow{5}{*}{ ugacagaagagagugagcac } \\
\hline & $\begin{array}{l}\text { osa-miR156d } \\
\text { MIMAT0000621 }\end{array}$ & & Chr2: 4512884-4513012 [-] & \\
\hline & $\begin{array}{l}\text { osa-miR156c-5p } \\
\text { MIMAT0000620 }\end{array}$ & & Chr1: 4665975-4666123 [+] & \\
\hline & $\begin{array}{l}\text { osa-miR156a } \\
\text { MIMAT0000618 }\end{array}$ & & Chr1: 22524147-22524246 [-] & \\
\hline & \begin{tabular}{|l|} 
osa-miR156h-5p \\
MIMAT0031156
\end{tabular} & & Chr8: 21491232-21491417 [+] & \\
\hline \multirow{3}{*}{ Cluster 2} & $\begin{array}{l}\text { osa-miR399b } \\
\text { MIMAT0000985 }\end{array}$ & \multirow{3}{*}{$\begin{array}{l}\text { MIPF0000015; } \\
\text { MIR399 }\end{array}$} & Chr2: 7664026-7664122 [-] & \multirow{3}{*}{ ugccaaaggagaauugcccug } \\
\hline & $\begin{array}{l}\text { osa-miR399a } \\
\text { MIMAT0000984 }\end{array}$ & & Chr1: 30478636-30478784 [+] & \\
\hline & $\begin{array}{l}\text { osa-miR399c } \\
\text { MIMAT0000986 }\end{array}$ & & Chr5: 26305938-26306047 [-] & \\
\hline Cluster 3 & $\begin{array}{l}\text { osa-miR171e-3p } \\
\text { MIMAT0001066 }\end{array}$ & $\begin{array}{l}\text { MIPF0000030; } \\
\text { MIR171_1 }\end{array}$ & Chr3: 1970487-1970605 [+] & ugauugagccgugccaauauc \\
\hline
\end{tabular}


Fatima Noor et al.

\begin{tabular}{|c|c|c|c|c|}
\hline & \multirow{2}{*}{\begin{tabular}{|l|} 
osa-miR171c-3p \\
MIMAT0001064 \\
osa-miR171d-3p \\
MIMAT0001065 \\
\end{tabular}} & & \multirow{2}{*}{$\begin{array}{c}\text { Chr4: } 31713472-31713570[-] \\
\text { Chr10: 21237313-21237447 [+] }\end{array}$} & \\
\hline & & & & \\
\hline \multirow{3}{*}{ Cluster 4} & $\begin{array}{l}\text { osa-miR164a } \\
\text { MIMAT0000633 }\end{array}$ & \multirow{3}{*}{$\begin{array}{l}\text { MIPF0000045; } \\
\text { MIR164 }\end{array}$} & Chr7: 28523341-28523496 [-] & \multirow{3}{*}{ uggagaagcagggcacgugca } \\
\hline & $\begin{array}{l}\text { osa-miR164f } \\
\text { MIMAT0001089 }\end{array}$ & & Chr5: 23343908-23344117 [+] & \\
\hline & \begin{tabular}{|l|} 
osa-miR164b \\
MIMAT0000634 \\
\end{tabular} & & Chr5: 15896163-15896271 [-] & \\
\hline \multirow{3}{*}{ Cluster 5} & $\begin{array}{l}\text { osa-miR167c-5p } \\
\text { MIMAT0000643 }\end{array}$ & \multirow{3}{*}{$\begin{array}{l}\text { MIPF0000023; } \\
\text { MIR167_1 }\end{array}$} & Chr3: 33130619-33130781 [+] & \multirow{3}{*}{ ugaagcugccagcaugaucua } \\
\hline & $\begin{array}{l}\text { osa-miR167a-5p } \\
\text { MIMAT0000641 }\end{array}$ & & Chr12: 25476808-25476948 [+] & \\
\hline & $\begin{array}{l}\text { osa-miR167b } \\
\text { MIMAT0000642 }\end{array}$ & & Chr3: 30546928-30547090 [-] & \\
\hline \multirow{3}{*}{ Cluster 6} & $\begin{array}{l}\text { osa-miR166b-3p } \\
\text { MIMAT0000636 }\end{array}$ & \multirow{3}{*}{$\begin{array}{l}\text { MIPF0000004; } \\
\text { MIR166 }\end{array}$} & Chr6: 30327084-30327289 [-] & \multirow{3}{*}{ Ucggaccaggcuucauucccc } \\
\hline & $\begin{array}{l}\text { osa-miR166a-3p } \\
\text { MIMAT0000635 }\end{array}$ & & Chr10: 19987135-19987279 [+] & \\
\hline & \begin{tabular}{|l|} 
osa-miR166c-3p \\
MIMAT0000637
\end{tabular} & & Chr3: 3487788-3487912 [+] & \\
\hline
\end{tabular}

Table-3. Summary of miRNAs grouped in clusters along with the members, genomic coordinates, gene families and the mature miRNA sequences in Zea mays

\begin{tabular}{|c|c|c|c|c|}
\hline Sr. No. & Members & $\begin{array}{|ll|}\begin{array}{l}\text { Gene } \\
\text { name }\end{array} & \text { family } \\
\end{array}$ & Genomic coordinates & Mature miRNA sequences \\
\hline \multirow{3}{*}{ Cluster 1} & \begin{tabular}{|l} 
Zma-miR399a-3p \\
MIMAT0001704
\end{tabular} & \multirow{3}{*}{$\begin{array}{l}\text { MIPF0000015; } \\
\text { MIR399 }\end{array}$} & chr4: 233617206-233617328 [-] & \multirow{3}{*}{ ugccaaaggagaauugcccug } \\
\hline & $\begin{array}{l}\text { zma-miR399h-3p } \\
\text { MIMAT0014023 }\end{array}$ & & chr5: 150363095-150363188 [+] & \\
\hline & $\begin{array}{l}\text { zma-miR399c-3p } \\
\text { MIMAT0001705 }\end{array}$ & & chr6: 163867932-163868138 [+] & \\
\hline \multirow{4}{*}{ Cluster 2} & $\begin{array}{l}\text { zma-miR156h-5p } \\
\text { MIMAT0001358 }\end{array}$ & \multirow{4}{*}{$\begin{array}{l}\text { MIPF0000008; } \\
\text { MIR156 }\end{array}$} & chr10: 129642870-129642984 [-] & \multirow{4}{*}{ ugacagaagagagugagcac } \\
\hline & $\begin{array}{l}\text { zma-miR156f-5p } \\
\text { MIMAT0001352 }\end{array}$ & & chr2: 185613978-185614144 [-] & \\
\hline & $\begin{array}{l}\text { zma-miR156e-5p } \\
\text { MIMAT0001356 }\end{array}$ & & chr2: 33005026-33005151 [+] & \\
\hline & \begin{tabular}{|l|} 
zma-miR1561-5p \\
MIMAT0013973
\end{tabular} & & chr5: 188595332-188595421 [-] & \\
\hline \multirow{2}{*}{ Cluster 3} & \begin{tabular}{|l|} 
zma-miR171j-3p \\
MIMAT0001738
\end{tabular} & \multirow{2}{*}{$\begin{array}{l}\text { MIPF0000030; } \\
\text { MIR171_1 }\end{array}$} & chr10: 59006552-59006680 [+] & \multirow{2}{*}{ ugauugagccgugccaauauc } \\
\hline & \begin{tabular}{|l|} 
zma-miR171i-3p \\
MIMAT0001740
\end{tabular} & & chr1: 278093492-278093595 [+] & \\
\hline \multirow{2}{*}{ Cluster 4} & $\begin{array}{l}\text { zma-miR167b-5p } \\
\text { MIMAT0001371 }\end{array}$ & \multirow{2}{*}{$\begin{array}{l}\text { MIPF0000023; } \\
\text { MIR167_1 }\end{array}$} & chr5: 7976369-7976495 [+] & \multirow{2}{*}{ Ugaagcugccagcaugaucua } \\
\hline & $\begin{array}{l}\text { zma-miR167c-5p } \\
\text { MIMAT0001373 }\end{array}$ & & chr5: 4414244-4414375 [+] & \\
\hline \multirow{2}{*}{ Cluster 5} & $\begin{array}{l}\text { zma-miR164c-5p } \\
\text { MIMAT0001367 }\end{array}$ & \multirow{2}{*}{$\begin{array}{l}\text { MIPF0000045; } \\
\text { MIR164 }\end{array}$} & chr6: 157477837-157478106 [+] & \multirow[t]{2}{*}{ Uggagaagcagggcacgugca } \\
\hline & \begin{tabular}{|l} 
zma-miR164a-5p \\
MIMAT0001364
\end{tabular} & & chr2: 223401157-223401308 [-] & \\
\hline
\end{tabular}


Fatima Noor et al.

\begin{tabular}{|l|l|l|}
\hline zma-miR164b-5p & & chr6: $145727415-145727542[+]$ \\
MIMAT0001366 &
\end{tabular}

Table-4. Summary of miRNAs grouped in clusters along with the members, genomic coordinates, gene families and the mature miRNA sequences in Brassica napus

\begin{tabular}{|c|c|c|c|c|}
\hline Sr. No. & Members & $\begin{array}{l}\text { Gene family } \\
\text { name }\end{array}$ & Genomic coordinates & Mature miRNA sequences \\
\hline \multirow{3}{*}{ Cluster 1} & \begin{tabular}{|l|} 
sbi-miR156a \\
MIMAT0001398
\end{tabular} & \multirow{3}{*}{$\begin{array}{l}\text { MIPF0000008; } \\
\text { MIR156 }\end{array}$} & chr4: 5326257-5326340 [-] & \multirow{3}{*}{ ugacagaagagagugagcac } \\
\hline & \begin{tabular}{|l|} 
sbi-miR156b \\
MIMAT0001400
\end{tabular} & & chr3: 3415906-3415989 [-] & \\
\hline & \begin{tabular}{|l|} 
sbi-miR156c \\
MIMAT0001399 \\
\end{tabular} & & chr3: 3416227-3416321 [-] & \\
\hline \multirow{2}{*}{ Cluster 2} & \begin{tabular}{|l|} 
sbi-miR399a \\
MIMAT0001437
\end{tabular} & \multirow{2}{*}{$\begin{array}{l}\text { MIPF0000015; } \\
\text { MIR399 }\end{array}$} & chr3: 61878757-61878892 [+] & \multirow[t]{2}{*}{ ugccaaaggagaauugcccug } \\
\hline & \begin{tabular}{|l|} 
sbi-miR399c \\
MIMAT0001438
\end{tabular} & & chr9: 55505804-55505933 [-] & \\
\hline \multirow{2}{*}{ Cluster 3} & $\begin{array}{l}\text { sbi-miR164e } \\
\text { MIMAT0011326 }\end{array}$ & \multirow{2}{*}{$\begin{array}{l}\text { MIPF0000045; } \\
\text { MIR164 }\end{array}$} & chr9: 44994962-44995166 [+] & \multirow[t]{2}{*}{ uggagaagcagggcacgugca } \\
\hline & \begin{tabular}{|l|} 
sbi-miR164a \\
MIMAT0001406
\end{tabular} & & chr9: 38911782-38911907 [-] & \\
\hline \multirow{2}{*}{ Cluster 4} & $\begin{array}{l}\text { sbi-miR171k } \\
\text { MIMAT0011344 }\end{array}$ & \multirow{2}{*}{$\begin{array}{l}\text { MIPF0000030; } \\
\text { MIR171_1 }\end{array}$} & chr6: 56762480-56762566 [-] & \multirow{2}{*}{ ugauugagccgugccaauauc } \\
\hline & \begin{tabular}{|l|} 
sbi-miR171i \\
MIMAT0011342
\end{tabular} & & chr1: 59668493-59668582 [-] & \\
\hline \multirow{2}{*}{ Cluster 5} & \begin{tabular}{|l|} 
sbi-miR394b \\
MIMAT0011349
\end{tabular} & \multirow{2}{*}{$\begin{array}{l}\text { MIPF0000100; } \\
\text { MIR394 }\end{array}$} & chr4: 62923468-62923544 [-] & \multirow{2}{*}{ uuggcauucuguccaccucc } \\
\hline & \begin{tabular}{|l|} 
sbi-miR394a \\
MIMAT0001427
\end{tabular} & & chr2: 66830969-66831078 [+] & \\
\hline \multirow{3}{*}{ Cluster 6} & $\begin{array}{l}\text { sbi-miR167b } \\
\text { MIMAT0001408 }\end{array}$ & \multirow{3}{*}{$\begin{array}{l}\text { MIPF0000023; } \\
\text { MIR167_1 }\end{array}$} & chr1: 7229987-7230184 [+] & \multirow{3}{*}{ ugaagcugccagcaugaucua } \\
\hline & \begin{tabular}{|l|} 
sbi-miR167i \\
MIMAT0011332
\end{tabular} & & chr8: 59223214-59223345 [+] & \\
\hline & \begin{tabular}{|l|} 
sbi-miR167a \\
MIMAT0001407
\end{tabular} & & chr1: 4354010-4354105 [+] & \\
\hline
\end{tabular}


Fatima Noor et al.

Table-5. Summary of miRNAs grouped in clusters along with the members, genomic coordinates, gene families and the mature miRNA sequences in Triticum aestivum

\begin{tabular}{|c|c|c|c|c|}
\hline Sr. No. & Members & $\begin{array}{c}\text { Gene family } \\
\text { name }\end{array}$ & Genomic coordinates & Mature miRNA sequences \\
\hline \multirow{4}{*}{ Cluster 1} & $\begin{array}{l}\text { bna-miR166a } \\
\text { MIMAT0005629 }\end{array}$ & \multirow{4}{*}{$\begin{array}{l}\text { MIPF0000004;M } \\
\text { IR166 }\end{array}$} & EM:DX911364: 697-831 [-] & \multirow{4}{*}{ ucggaccaggcuucauucccc } \\
\hline & $\begin{array}{l}\text { bna-miR166c } \\
\text { MIMAT0005631 }\end{array}$ & & EM:AC189591: 82389-82516 [+] & \\
\hline & $\begin{array}{l}\text { bna-miR166b } \\
\text { MIMAT0005630 }\end{array}$ & & $\begin{array}{c}\text { EM:BH423978: 306-423 } \\
{[+] \text { EM:BH680789: 165-282 [-] }}\end{array}$ & \\
\hline & $\begin{array}{l}\text { bna-miR166d } \\
\text { MIMAT0005632 }\end{array}$ & & EM:AC189313: 1397-1524 [+] & \\
\hline \multirow{2}{*}{ Cluster 2} & $\begin{array}{l}\text { bna-miR394b } \\
\text { MIMAT0023635 }\end{array}$ & \multirow{2}{*}{$\begin{array}{l}\text { MIPF0000100;M } \\
\text { IR394 }\end{array}$} & EM:AC189295: 85927-86038 [+] & \multirow{2}{*}{ Uuggcauucuguccaccucc } \\
\hline & $\begin{array}{l}\text { bna-miR394a } \\
\text { MIMAT0023634 }\end{array}$ & & EM:AC189318: 31776-31891 [+] & \\
\hline \multirow{2}{*}{ Cluster 3} & $\begin{array}{l}\text { bna-miR156e } \\
\text { MIMAT0023614 }\end{array}$ & \multirow{2}{*}{$\begin{array}{l}\text { MIPF0000008; M } \\
\quad \text { IR156 }\end{array}$} & $\begin{array}{c}\text { EM:AC189375: 124714-124832 [+] } \\
\text { EM:DU831758: 290-408 [-] }\end{array}$ & \multirow{2}{*}{ Ugacagaagagagugagcac } \\
\hline & $\begin{array}{l}\text { bna-miR156f } \\
\text { MIMAT0023615 }\end{array}$ & & EM:CV432746: 228-337 [+] & \\
\hline
\end{tabular}

\section{Identification of Inter-specific homologous mature miRNAs}

The multiple aligned sequences were subjected phylogenetic analyses (Figure 8). The analysis comprised of 73 nucleotide sequences, and the final dataset was shown to contain 26 positions which were grouped into 7 clusters. It was interesting to see that one miRNA- UGACAGAAGAGAGUGAGCAC was conserved in Glycine max, Oryza sativa, Zea mays, Sorghum bicolor, and Brassica napus. Another miRNA-UGCCAAAGGAGAAUUGCCCUG was shown to be conserved in Glycine max, Oryza sativa, Zea mays, and Sorghum Bicolor. While another miRNA-UGAUUGAGCCGUGCCAAUAUC was shown to be conserved in Glycine max, Oryza sativa, Zea mays, Sorghum bicolor, and Triticum aestivum. The miRNA- UGAAGCUGCCAGCAUGAUCUA was found to be conserved in Glycine max, Oryza sativa, Zea mays, Sorghum bicolor, Brassica napus, and Triticum aestivum. The miRNAUGGAGAAGCAGGGCACGUGCA was found to be conserved in Glycine max, Oryza sativa, Zea mays, Sorghum bicolor, Brassica napus, and Triticum aestivum. The miRNAUUGGCAUUCUGUCCACCUCC was found to be conserved in Glycine max, Sorghum bicolor, and Brassica napus. While, the miRNAUCGGACCAGGCUUCAUUCCCC was shown to be conserved in Glycine max, Zea mays, Sorghum bicolor, and Brassica napus.

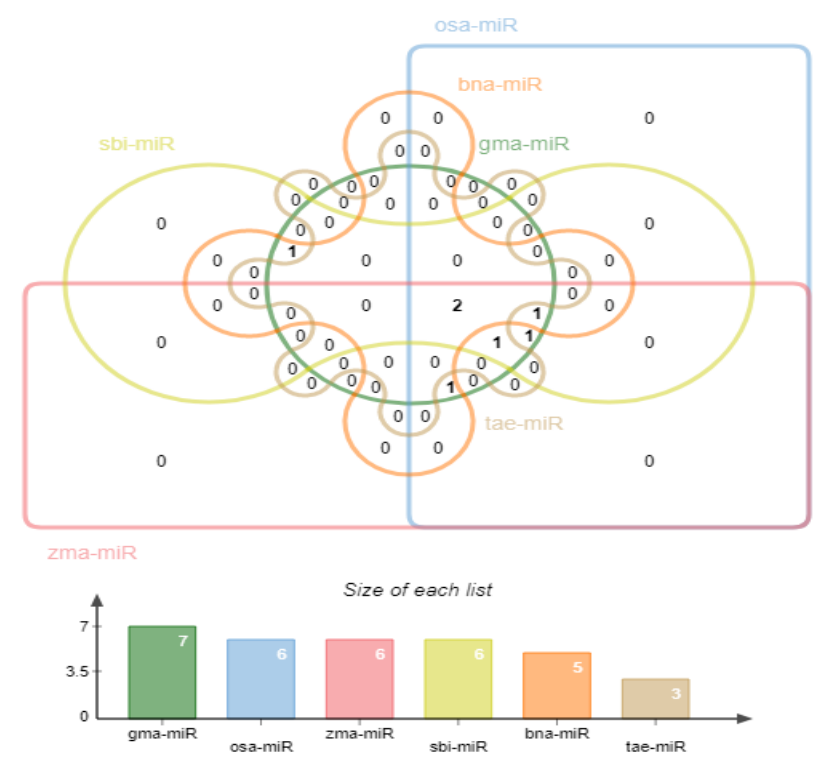

Figure-7. Representation of overlapped miRNA in Glycine max, Oryza sativa, Zea mays, Sorghum bicolor, Brassica napus, and Triticum aestivum 


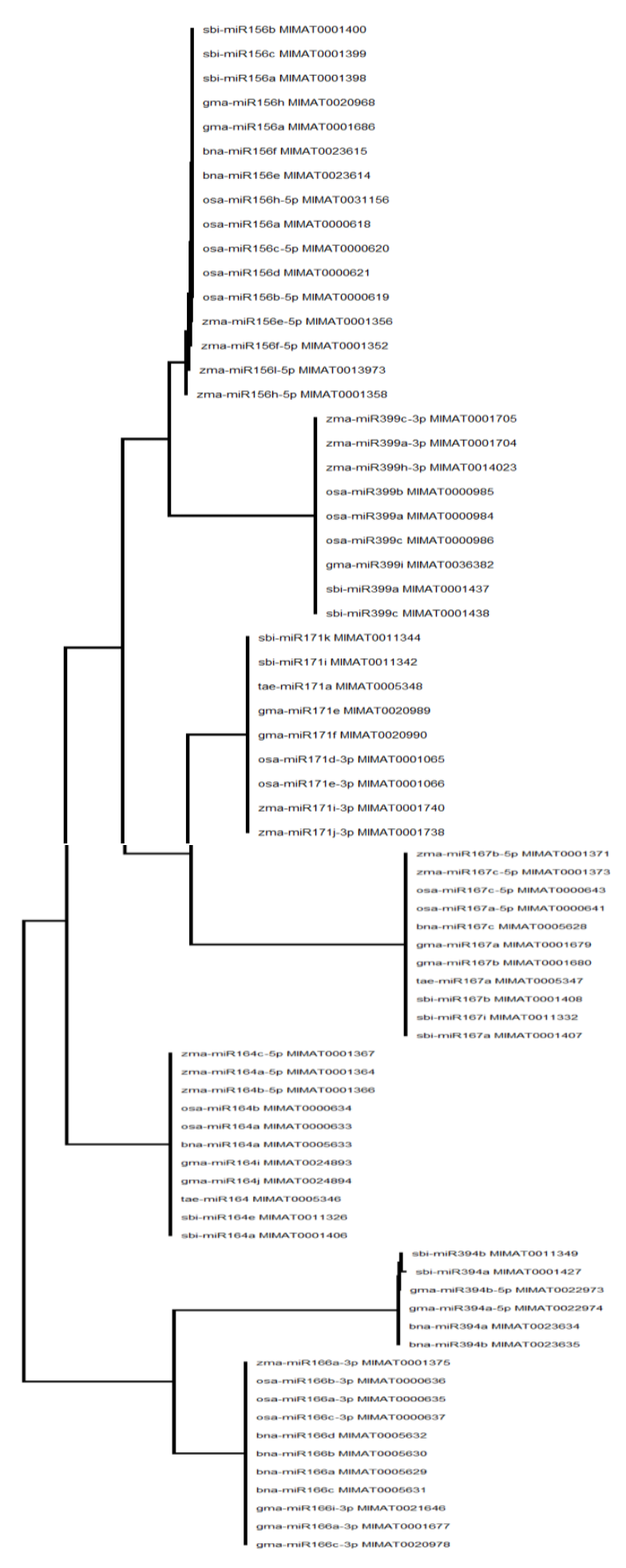

Figure-8. Representation of evolutionary relationship among Glycine max, Oryza sativa, Zea mays, Sorghum bicolor, Brassica napus, and Triticum aestivum based on 73 sequences.

\section{Discussion}

It is shown that miRNAs are involved the posttranscriptional gene expression (Zhang et al., 2006a). In spite of the fact that miRNA is common in animals, some miRNA clusters are found to be conserved in plants (Sunkar and Zhu, 2004, Guddeti et al., 2005, Zhang et al., 2007, Talmor- Neiman et al., 2006). However, insertion, deletion and duplication events in miRNA sequences suggested that evolutionary conserved clusters are present in plants. However, it has been estimated that gene duplication events occur more frequently in eukaryotic genomes (Lynch and Conery, 2000) and particularly in flowering plants (Blanc and Wolfe, 2004, Cui et al., 2006). It has been investigated that plant comprises more non-conserved clusters when compared to the conserved clusters (Fahlgren et al., 2007, Rajagopalan et al., 2006, Sunkar et al., 2008).

Several in-silico and in-vitro studies have identified conserved miRNA in various bioenergy crops, but none deciphered the conserved identical miRNA sequences in the group of bioenergy crops. The present study was focused to identify the intra-specific and inter-specific conserved miRNAs in six bioenergy crops. Through computational and experimental identification, miRBase is considered as one of the main storehouses to collect miRNA genes. The present study elucidated 7 miRNA families that found to be conserved in six bioenergy crops. The conserved miRNA clusters were shown to belong miR166, miR399, miR156, miR171, miR164, miR167, and miR394 families indicated that due to genomic duplication event the ancestral clusters might have been originated.

The miR156 represents an evolutionary conserved miRNA which indicated that it is common in plant species. Interestingly, the bioenergy crops overexpressing miR156 were shown to increase the plant biomass and altered lignin content and composition (Fu et al., 2012, Rubinelli et al., 2013, Schwab et al., 2005). The miR156 has also found associated with the phase transition, in the plant development, formation of floral meristem, and morphology of immature leaves and cell wall. Besides, miR156 has shown to be involved in abiotic stress responses including drought and low-nitrogen in bioenergy crops (Ferreira et al., 2012, Khraiwesh et al., 2012). A higher expression level of miR156 led to a shortened length of internode and hence the overall plant biomass decreased (Fu et al., 2012). Both of the 
miR166 and miR167 are involved in metabolism, morphology, and development of Zea mays and Sorghum bicolor (Wei et al., 2009). Moreover, miR166 and miR167 are also involved in the early development of plant, hence could have potential application in abiotic stress, biofuel yield, bio confinement, and recalcitrance (Trumbo et al., 2015). The miR164 is another evolutionary conserved miRNA which is found associated with the metabolic processes, drought response, early development (Wei et al., 2009), regulation of lateral rooting. Hence, it could be another target of metabolic engineering to counteract stress and to enhance plant biomass production (Wei et al., 2009). In Sorghum bicolor, miR399 may have potential application in abiotic stress. During water deprivation, miR399 was upregulated and showed a positive stress response (Calviño et al., 2011, Katiyar et al., 2012, Paterson et al., 2009). In Glycine Max, miR399 could be an engineering target to control the phosphate regulation (Sun, 2012). In Zea mays, miR399 has shown to involved regulating the morphogenesis and embryonic development in the grain ( $\mathrm{Li}$ et al., 2016). In Oryza sativa, miR399 has shown to be involved in phosphate signaling (Fang et al., 2009). In Sorghum bicolor, miR171 is associated with the stress responses and in the process of morphological development (Ram and Sharma, 2013). While the upregulation of miR171 have shown to be involved in abiotic stress and in floral development in Oryza Sativa (Zhou et al., 2010). Further in vitro studied are required on miR399, miR171, and miR394 for their exploitation as future targets of metabolic engineering to counteract the abiotic stress and to enhance biomass production of the bioenergy crops.

\section{Conclusion}

The study was focused on identification of conserved miRNAs in selected bioenergy crops as future targets of metabolic engineering to improve the biomass productivity. Based on phylogenetic analyses, conserved miRNA clusters were shown to belong to miR166, miR399, miR156, miR171, miR164, miR167, and miR394 families, while these families can be used as genetic engineering targets. Such studies can further be extended to other crops of agricultural and environmental importance to identify conserved miRNAs to understand their physiological roles and evolutionary relationships.
Disclaimer: None.

Conflict of Interest: None. Source of Funding: None.

\section{References}

Ahmad MS, Mehmood MA, Al Ayed OS, Ye G, Luo H, Ibrahim M, Rashid U, Nehdi IA and Qadir G, 2017. Kinetic analyses and pyrolytic behavior of Para grass (Urochloa mutica) for its bioenergy potential. Bioresourc. Technol. 224: 708-713.

Bardou P, Mariette J, Escudié F, Djemiel C and Klopp C, 2014. Jvenn: an interactive Venn diagram viewer. BMC Bioinform. 15(1): 1-7.

Blanc $G$ and Wolfe KH, 2004. Widespread paleopolyploidy in model plant species inferred from age distributions of duplicate genes. Plant Cell. 16(7): 1667-1678.

Calviño M, Bruggmann R and Messing J, 2011. Characterization of the small RNA component of the transcriptome from grain and sweet sorghum stems. BMC Genom. 12(1): 356.

Chenna R, Sugawara H, Koike T, Lopez R, Gibson TJ, Higgins DG and Thompson JD, 2003. Multiple sequence alignment with the Clustal series of programs. Nucleic Acids Res. 31: 3497-3500.

Chitwood DH, Nogueira FT, Howell MD, Montgomery TA, Carrington JC and Timmermans MC, 2009. Pattern formation via small RNA mobility. Genes Dev. 23(5): 549-554.

Cui L, Wall PK, Leebens-Mack JH, Lindsay BG, Soltis DE, Doyle JJ and Barakat A, 2006. Widespread genome duplications throughout the history of flowering plants. Genome Res. 16(6): 738-749.

Fahlgren N, Howell MD, Kasschau KD, Chapman EJ, Sullivan CM, Cumbie JS and Dangl JL, 2007. High-throughput sequencing of Arabidopsis microRNAs: evidence for frequent birth and death of MIRNA genes. PloS ONE. 2(2): e219.

Fang Z, Shao C, Meng Y, Wu P and Chen M, 2009. Phosphate signaling in Arabidopsis and Oryza sativa. Plant Sci. 176(2): 170-180.

Ferreira TH, Gentile A, Vilela RD, Costa GGL, Dias LI, Endres L and Menossi M, 2012. microRNAs associated with drought response in the bioenergy crop sugarcane (Saccharum spp.). PLoS ONE. 7(10): e46703.

Fu C, Sunkar R, Zhou C, Shen H, Zhang JY, Matts J and Tang Y, 2012. Overexpression of miR156 in switchgrass (Panicum virgatum L.) results in 
various morphological alterations and leads to improved biomass production. Plant Biotechnol. J. 10(4): 443-452.

Gokcol C, Dursun B, Alboyaci B and Sunan E, 2009. Importance of biomass energy as alternative to other sources in Turkey. Energy Policy. 37(2): 424-431..

Griffiths-Jones S, Saini HK, Van Dongen S and Enright AJ, 2007. miRBase: tools for microRNA genomics. Nucleic Acids Res. 36(1): D154-D158.

Guddeti S, Li AL, Leseberg CH, Hui K, Li XG, Zhai WX and Long M, 2005. Molecular evolution of the rice miR395 gene family. Cell Res. 15(8): 631638.

Guo HS, Xie Q, Fei JF and Chua NH, 2005. MicroRNA directs mRNA cleavage of the transcription factor NAC1 to downregulate auxin signals for Arabidopsis lateral root development. Plant Cell. 17(5): 1376-1386.

Joshi R, Gupta P, Singla-Pareek SL and Pareek AJ, 2017. Biomass production and salinity response in plants: role of MicroRNAs. Indian J. Plant Physiol. 22: 448-457.

Katiyar A, Smita S, Chinnusamy V, Pandey DM and Bansal K, 2012. Identification of miRNAs in sorghum by using bioinformatics approach. Plant Signal. Behavior. 7(2): 246-259.

Khraiwesh B, Zhu JK and Zhu J, 2012. Role of miRNAs and siRNAs in biotic and abiotic stress responses of plants. Biochim. Biophys. Acta. 1819(2): 137-148.

Li D, Liu Z, Gao L, Wang L, Gao M, Jiao Z and Yao L, 2016. Genome-Wide Identification and Characterization of microRNAs in Developing Grains of Zea mays L. PloS ONE. 11(4): e0153168.

Lynch M and Conery JS, 2000. The evolutionary fate and consequences of duplicate genes. Science. 290(5494): 1151-1155.

Mehmood MA, Ahmad MS, Liu Q, Liu CG, Tahir MH, Aloqbi AA, Tarbiah NI, Alsufiani HM and Gull M, 2019. Helianthus tuberosus as a promising feedstock for bioenergy and chemicals appraised through pyrolysis, kinetics, and TGFTIR-MS based study. Energy Convers. Manage. 194: 37-45.

Mehmood MA, Ibrahim M, Rashid U, Nawaz M, Ali $\mathrm{S}$ and Hussain A, 2017. Biomass production for bioenergy using marginal lands. Sustain. Prod. Consum. 9: 3-21.
Nogueira FT, Madi S, Chitwood DH, Juarez MT and Timmermans MC, 2007. Two small regulatory RNAs establish opposing fates of a developmental axis. Genes Dev. 21(7): 750-755.

Paterson AH, Bowers JE, Bruggmann R, Dubchak I, Grimwood J, Gundlach H and Poliakov A, 2009. The Sorghum bicolor genome and the diversification of grasses. Nature. 457(7229): 551556.

Rajagopalan R, Vaucheret H, Trejo J and Bartel DP, 2006. A diverse and evolutionarily fluid set of microRNAs in Arabidopsis thaliana. Genes Dev. 20: 3407-3425.

Ram G and Sharma AD, 2013. In silico analysis of putative miRNAs and their target genes in sorghum (Sorghum bicolor). Int. J. Bioinfom. Res. Appl. 9(4), 349-364.

Rubinelli P M, Chuck G, Li X and Meilan R, 2013. Constitutive expression of the Corngrass 1 microRNA in poplar affects plant architecture and stem lignin content and composition. Biomass Bioener. 54: 312-321.

Rubio-Somoza I, Cuperus JT, Weigel D and Carrington JC, 2009. Regulation and functional specialization of small RNA-target nodes during plant development. Curr. Opin. Plant Biol. 12(5): 622-627.

Ruiz-Ferrer V and Voinnet O, 2009. Roles of plant small RNAs in biotic stress responses. Ann. Rev. Plant Biol. 60: 485-510.

Schwab R, Palatnik JF, Riester M, Schommer C, Schmid M and Weigel D, 2005. Specific effects of microRNAs on the plant transcriptome. Dev. Cell. 8(4): 517-527.

Shahid A, Malik S, Zhu H, Xu J, Nawaz MZ, Nawaz S, Mehmood MA and Alam MA, 2020. Cultivating microalgae in wastewater for biomass production, pollutant removal, and atmospheric carbon mitigation; a review. Sci. Total Environ. 704: 135303.

Shukla LI, Chinnusamy V and Sunkar R, 2008. The role of microRNAs and other endogenous small RNAs in plant stress responses. Biochim. Biophys. Acta. 1779(11): 743-748.

Sims RE, Hastings A, Schlamadinger B, Taylor G and Smith P, 2006. Energy crops: current status and future prospects. Glob. Change Biol. 12(11): 2054-2076.

Sun G, 2012. MicroRNAs and their diverse functions in plants. Plant Mole. Biol. 80(1), 17-36. 
Sunkar R, Zhou X, Zheng Y, Zhang W and Zhu JK, 2008. Identification of novel and candidate miRNAs in rice by high throughput sequencing. BMC Plant Biol. 8(1):1-7.

Sunkar R and Zhu JK, 2004. Novel and stressregulated microRNAs and other small RNAs from Arabidopsis. Plant Cell. 16(8):2001-19.

Talmor- Neiman M, Stav R, Frank W, Voss B and Arazi T, 2006. Novel micro- RNAs and intermediates of micro- RNA biogenesis from moss. Plant J. 47(1): 25-37.

Trumbo JL, Zhang B and Stewart Jr CN, 2015. Manipulating micro RNA s for improved biomass and biofuels from plant feedstocks. Plant Biotechnol. J. 13(3): 337-354.

Wei B, Cai T, Zhang R, Li A, Huo N, Li S and Qi Y, 2009. Novel microRNAs uncovered by deep sequencing of small RNA transcriptomes in bread wheat (Triticum aestivum L.) and Brachypodium distachyon (L.) Beauv. Funct. Integr. Genomics. 9(4): 499.

Wright L, 2006. Worldwide commercial development of bioenergy with a focus on energy crop-based projects. Biomass Bioener. 30(8-9): 706-714.

Ye G, Luo H, Ren Z, Ahmad MS, Liu CG and Tawab A, 2018. Evaluating the bioenergy potential of Chinese Liquor-industry waste through pyrolysis, thermogravimetric, kinetics and evolved gas analyses. Energy Convers. Manag. 163: 13-21.

Yuan JS, Tiller KH, Al-Ahmad H, Stewart NR and Stewart Jr CN, 2008. Plants to power: bioenergy to fuel the future. Trends Plant Sci. 13(8): 421429.

Zhang B, Pan X, Cannon CH, Cobb GP and Anderson TA, 2006. Conservation and divergence of plant microRNA genes. Plant J. 46(2): 243-259.

Zhang B, Pan X, Wang Q, Cobb GP and Anderson TA, 2006. Computational identification of microRNAs and their targets. Comput. Biol. Chem. 30(6): 395407.

Zhang B, Wang Q, Wang K, Pan X, Liu F, Guo T and Anderson TA, 2007. Identification of cotton microRNAs and their targets. Genes. 397(1-2): 26-37.

Zhao CZ, Xia H, Frazier TP, Yao YY, Bi YP, Li AQ and Wang XJ, 2010. Deep sequencing identifies novel and conserved microRNAs in peanuts (Arachis hypogaea L.). BMC Plant Biol. 10(1): 3.

Zhou L, Liu Y, Liu Z, Kong D, Duan M and Luo L, 2010. Genome-wide identification and analysis of drought-responsive microRNAs in Oryza sativa. J. Exper. Bot. 61(15): 4157-4168

\section{Contribution of Authors}

Noor F: Performed all analysis of current study

Alshamrani R: Helped in data curation

Gull M: Manuscript editing and write up

Mehmood MA: Litersture review and manuscript editing

Aslam S: Supervised the research, final reading and approval of manuscript 